Thomas Duzia | Norbert Bogusch

\title{
Basiswissen Bauphysik
}

Grundlagen des Wärme- und Feuchteschutzes

3., überarbeitete und erweiterte Auflage

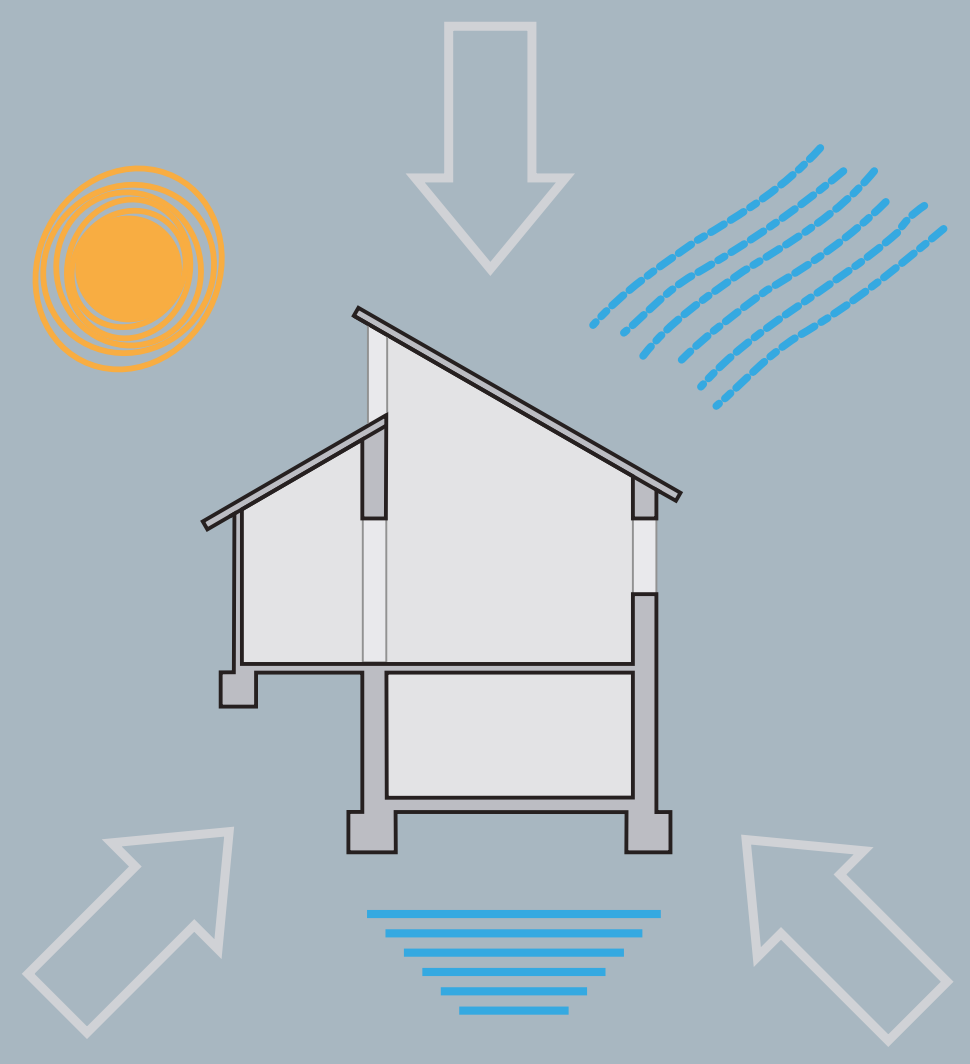

Fraunhofer IRB Verlag 
Dr.-Ing. Thomas Duzia

Dipl.-Ing. (FH) Norbert Bogusch

Basiswissen Bauphysik 
Dr.-Ing. Thomas Duzia

Dipl.-Ing. (FH) Norbert Bogusch

\section{Basiswissen Bauphysik}

3., überarbeitete und erweiterte Auflage

Fraunhofer IRB Verlag 
Bibliografische Information der Deutschen Nationalbibliothek:

Die Deutsche Nationalbibliothek verzeichnet diese Publikation in der Deutschen Nationalbibliografie; detaillierte bibliografische Daten sind im Internet über www.dnb.de abrufbar.

ISBN (Print): $\quad$ 978-3-7388-0419-5

ISBN (E-Book): 978-3-7388-0420-1

Satz, Herstellung, Layout: Gabriele Wicker

Umschlaggestaltung: Martin Kjer

Druck: Offizin Scheufele Druck und Medien GmbH + Co.KG, Stuttgart

Die hier zitierten Normen sind mit Erlaubnis des DIN Deutsches Institut für Normung e.V. wiedergegeben. Maßgebend für das Anwenden einer Norm ist deren Fassung mit dem neuesten Ausgabedatum, die bei der Beuth Verlag GmbH, Burggrafenstraße 6, 10787 Berlin, erhältlich ist.

Alle Rechte vorbehalten.

Dieses Werk ist einschließlich aller seiner Teile urheberrechtlich geschützt. Jede Verwertung, die über die engen Grenzen des Urheberrechtsgesetzes hinausgeht, ist ohne schriftliche Zustimmung des Fraunhofer IRB Verlages unzulässig und strafbar. Dies gilt insbesondere für Vervielfältigungen, Übersetzungen, Mikroverfilmungen sowie die Speicherung in elektronischen Systemen.

Die Wiedergabe von Warenbezeichnungen und Handelsnamen in diesem Buch berechtigt nicht zu der Annahme, dass solche Bezeichnungen im Sinne der Warenzeichen- und Markenschutz-Gesetzgebung als frei zu betrachten wären und deshalb von jedermann benutzt werden dürften.

Sollte in diesem Werk direkt oder indirekt auf Gesetze, Vorschriften oder Richtlinien (z.B. DIN, VDI, VDE) Bezug genommen oder aus innen zitiert werden, kann der Verlag keine Gewähr für Richtigkeit, Vollständigkeit oder Aktualität übernehmen. Es empfiehlt sich, gegebenenfalls für die eigenen Arbeiten die vollständigen Vorschriften oder Richtlinien in der jeweils gültigen Fassung hinzuzuziehen.

(C) Fraunhofer IRB Verlag, 2020

Fraunhofer-Informationszentrum Raum und Bau IRB

Nobelstraße 12, 70569 Stuttgart

Telefon $+49711970-2500$

Telefax $+49711970-2508$

irb@irb.fraunhofer.de

www.baufachinformation.de 


\section{Vorwort}

Klimaschutz und Begrenzung des globalen Temperaturanstiegs auf möglichst »unter 2 Grad C « steht in Europa und in Deutschland auf der politischen Top-Agenda und beeinflusst den Ausgang von Wahlen wie nie zuvor. Namhafte Großstädte wie etwa Köln beschließen den Klimanotstand. Der alarmierende Tenor wissenschaftlicher Studien für den weltweiten Klimarat (IPCC) nimmt seit Jahren zu. Der Jugend platzt der Kragen (Fridays4Future), weil ihr die Zukunft gestohlen wird. Mehr als 23000 Wissenschaftler von Scientist4Future unterstützen sie. Eine derartige Einmütigkeit und Solidaritätserklärung gab es noch nie in der deutschsprachigen Wissenschaft. Die Extremwetterereignisse der letzten Jahre signalisieren, was wissenschaftlich nicht mehr bestritten werden kann: Der Klimawandel ist sichtbar, fühlbar, messbar und er beschleunigt sich. Nur wenige Ignoranten und Lobbyisten leugnen dies.

Die ambitionierteste Energiewende der Welt hat Deutschland in den Jahren 2010/2011 mit einem quantifizierten Zielkanon für 2050 beschlossen. Ein Jahrhundertprojekt ohne Vorbild: Halbierung des Energieverbrauchs, raus aus Kohle, Öl, Erdgas und Uran! Einen solchen staatlich forcierten Strukturwandel hat es ebenfalls noch nie gegeben. Bei der Stromwende gibt es wurden beeindruckende Fortschritte gemacht: $47 \%$ der Nettostromerzeugung stammten inzwischen im 1. Halbjahr 2019 aus erneuerbaren Quellen. Einen gleichzeitigen Atomausstieg (bis 2022) und ein Kohle-Ausstiegsjahr (bis spätestens 2038) hat noch kein vergleichbares Industrieland beschlossen. Diese Erfolge sollte man nicht kleinreden. Aber der anspruchsvollste und schwierigste Teil der Energiewende liegt noch vor uns. Vor allem bei der energetischen Sanierung und Dekarbonisierung des Gebäudebestands steht die deutsche Energiewende- und Klimapolitik vor der Herausforderung eines epochalen Gemeinschaftswerkes - vielleicht nicht so spektakulär wie der nachhaltige Umbau des Verkehrssystems, dafür aber umso komplexer.

Im Klimaschutzplan hat die Bundesregierung 2015 Sektorziele für 2030 beschlossen. Für den Gebäudebereich bedeutet dies eine Reduktion um fast $67 \%$ (d. h. etwa um 70 Mio t $\mathrm{CO}_{2}$ equiv.) im Vergleich zu 2014, nur dann wird bis 2050 ein nahezu klimaneutraler Gebäudestand erreicht. Es gibt Szenarien, die zeigen, dass das durch ein Zukunftsinvestitionsprogramm wirtschaftlich machbar wäre. Notwendig ist eine milliardenschwere, kontinuierliche jährliche Anschubfinanzierung, deren volkswirtschaftlichen Effekte überwiegend positiv sind. Was bei solchen gesamtwirtschaftlichen Analysen aber oft vernachlässigt ist wird, sind die nur scheinbar einfachen Fragen: Wer setzt das alles um? Gibt es freie Baukapazitäten? Brauchen wir nicht zuallererst eine Qualifikationsoffensive? Ohne einen gewaltigen Schub bei Fachkräften und bei der baufachlichen Fundierung von Breiten- und Systemwissen wird es keinen dekarbonisierten Gebäudebereich geben.

Hier kommt der neu aufgelegte Band »Basiswissen Bauphysik « genau zum richtigen Zeitpunkt. Es ist ein perfektes Nachschlagewerk und wie ein Lexikon der Bauphysik differenziert gegliedert und nutzbar. Es ist so geschrieben, dass nicht nur Fachleute, Ingenieure und Architekten, Wissenslücken auffüllen können. Selbst Bauherren oder Investoren können nach der Lektüre einzelner Kapitel verstehen, wie energetischer Wärmeschutz funktioniert, warum er notwendig ist, wie Brandgefährdung und Schimmelbildung vermieden werden können und warum - angesichts der Zunahme der Häufigkeit und Intensität von Hitzewellen - auch in unseren Breiten der sommerliche Wärmeschutz an Bedeutung gewinnt. 
Die fachlichen Anforderungen und die Systemintegration bei der beschleunigten Implementierung der Energiewende im Gebäudebereich werden in Zukunft sprunghaft zunehmen. Sektorkopplung durch grünen Strom und nachhaltige Mobilität werden als neue Herausforderungen dazukommen. Daher ist dem Band heute und in Zukunft eine starke Verbreitung zu wünschen. Die nächste Auflage in Richtung »Zukünftige Anforderungen« sollte bereits geplant werden.

Prof. Dr. Peter Hennicke

Ehemaliger Präsident des Wuppertal Instituts; Full Member of the Club of Rome

\section{Vorwort zur zweiten Auflage}

Die neue Energieeinsparverordnung vom Mai 2014 reiht sich in eine ganze Folge an gesetzlichen Vorgaben ein, die 1977 mit der ersten Wärmeschutzverordnung begann. Die Bundesregierung verbindet mit diesen Vorgaben das langfristige Ziel eines »nahezu klimaneutralen Gebäudebestands « im Jahr 2050. Von diesem hehren Ziel ist man allerdings trotz der Verordnungen noch weit entfernt. Derzeit ist allein die Beheizung des Gebäudebestands für etwa ein Drittel des deutschen Gesamtenergieverbrauchs - zu großen Teilen nicht »klimaneutral « gewonnene Energie - verantwortlich. Dies zeigt, welche Bedeutung in den nächsten Jahren und Jahrzehnten der zum Bereich der Bauphysik zählende Wärmeschutz einnehmen wird. Daher ist es nur folgerichtig, dass diesem Thema im vorliegenden Buch eine dominierende Stellung zugeteilt wird.

Des Weiteren gehen die Autoren unter Berücksichtigung der novellierten DIN 4108-2 auf den sommerlichen Wärmeschutz ein. Dieser gewinnt zunehmend an Bedeutung, nicht zuletzt bedingt durch die in der modernen Architektur häufig anzutreffenden großzügig dimensionierten Fensterflächen, die einen hohen solaren Eintrag zur Folge haben können. Hinzu kommt die mitunter geringe Wärmekapazität solcher Gebäude, die eine sommerliche Überhitzung in hohem Maße fördert. Ein weiterer Fokus des vorliegenden Buches ist dem Feuchteschutz gewidmet. Auch hier haben die Autoren die richtige Wahl getroffen, da Feuchteschäden jeglicher Couleur in deutschen Gebäuden sehr häufig anzutreffen sind. Abgerundet werden die genannten Themen durch das Aufzeigen verschiedener Schadensbilder, mit denen die beiden Autoren als anerkannte Sachverständige sehr häufig konfrontiert werden.

Diese Hintergründe verdeutlichen, dass möglichst viele am Bau Beteiligte über ein bauphysikalisches Basiswissen verfügen sollten. In diese Lücke stößt das vorliegende Buch, in dem die Grundlagen des Wärmeschutzes, der aktuellen Energieeinsparverordnung sowie das Thema Feuchteschutz aufbereitet dargestellt werden. Damit bietet das Buch eine hervorragende grundlegende Orientierung im Bereich der Bauphysik, sowohl für Anfänger als auch Fortgeschrittene. Dies ist notwendig, weil die immer größer werdende Zahl zu berücksichtigender Normen und anderer Vorgaben nur schwer zu durchdringen ist. Zudem hat sich der Stand von Wissenschaft und Technik kontinuierlich weiterentwickelt, was Planer selbstverständlich berücksichtigen müssen. 
Zielgruppe des Buches sind deshalb sowohl Einsteiger, die sich einen bauphysikalischen Überblick verschaffen wollen als auch Bauingenieuren und Architekten, deren bauphysikalische Ausbildung an den Universitäten und Fachhochschulen eine Weile zurückliegt und die sich beruflich weiterbilden wollen. Darüber hinaus sollen alle Leser herzlich eingeladen sein, diese »Basis« als Ausgangspunkt für einen vertiefenden Einstieg in die Bauphysik zu verstehen.

Jun.-Prof. Dr.-Ing. Conrad Völker

TU Kaiserslautern

\section{Vorwort zur ersten Auflage}

Als die Autoren mich gebeten haben, ein Vorwort zu ihrer Veröffentlichung »Basiswissen Bauphysik « zu verfassen, habe ich spontan zugesagt. Der Grund dafür liegt nicht zuletzt darin, dass sich das Bauwesen in den letzten Jahrzehnten grundlegend verändert hat und damit neue Maßstäbe und Anforderungen gestellt werden, denen die Baupraxis nicht immer zeitnah gerecht werden kann. Die Ursachen dafür sind in erster Linie darin zu finden, dass sich auf der einen Seite die Richtlinien, Vorschriften und Normen immer schneller und öfter verändern und dass auf der anderen Seite die Entwicklung neuer Baustoffe, Instandsetzungssysteme und Verfahrenstechniken ebenfalls rasant zugenommen hat. Es ist daher nicht verwunderlich, dass die Ausbildung in Lehre und Praxis diesem Tempo häufig nicht schritthalten kann. Um diese Lücken zu schließen, ist die berufliche Weiterbildung gefordert, ohne die der Wissensstand der Technik nicht gehalten werden kann.

Wenn sich nun zwei erfahrene Sachverständige und Architekten des Themas Bauphysik mit dem Schwerpunkt des Wärmeschutzes unter Berücksichtigung des Einflusses der Feuchtigkeit annehmen und ein für die eigenen Berufskollegen bestimmtes Fachbuch erarbeiten, ist dies zweifelsohne ein Schritt in die richtige Richtung. In verständlicher Form werden in elf Kapiteln die Anforderungen der EnEV an den Wärmeschutz von Gebäuden vermittelt. Dabei werden nicht nur die gesetzlichen oder normativen Vorschriften berücksichtigt, sondern auch die Grundlagen und Grundbegriffe des Wärmeschutzes definiert und erläutert. Die Darstellung von Problemen mit schadensrelevanter Bedeutung kommt dabei ebenfalls nicht zu kurz. Am Wichtigsten ist jedoch, dass die Probleme mit angemessenen Lösungsvorschlägen kombiniert werden. Besonders hilfreich sind dabei die zahlreichen Tabellen und Abbildungen, die die notwendige Anschaulichkeit und Verständlichkeit gewährleisten.

Das Buch schließt eine Lücke in der Fachliteratur für die Ausbildung und Weiterbildung von Planern, Sachverständigen und Fachberatern.

\section{Prof. Dr. Helmut Weber}




\section{Anmerkung der Autoren}

Die einzelnen Kapitel sind nach den Kompetenzbereichen von jeweils einem Autor verfasst worden. Norbert Bogusch ist Verfasser der Kapitel 3.1, 3.2, 3.5, 4.4.1 und 7. Verfasser der Kapitel 1, 2, 3.3, 3.4, 6, 8, 9 und 11 ist Thomas Duzia. Die Kapitel 4, 5, 8.9.1, 8.9.2, 8.9.3 und 10 entstanden gemeinschaftlich.

Selbstverständlich sind beide Autoren zusätzlich für den gesamten zu vermittelnden Stoff verantwortlich, da die Ausführung aus einer gemeinschaftlichen Konzeption entstanden ist.

Die Überarbeitungen zur zweiten und dritten Auflage wurden von Dr.-Ing. Thomas Duzia vorgenommen. 


\section{Inhaltsverzeichnis}

$1 \quad$ Die historische Entwicklung des Wärme- und Feuchteschutzes _ 13

$2 \quad$ Übersicht geltender Regelwerke zum Wärme- und Feuchteschutz 17

2.1 DIN 4108 - Wärmeschutz und Energie-Einsparung in Gebäuden 18

2.2 Wärmeschutzverordnung - WSchV 19

$\begin{array}{ll}2.3 \quad \text { Energieeinsparverordnung - EnEV } & 19\end{array}$

2.3.1 Verbrauchsausweis zur EnEV $\quad 21$

$\begin{array}{lll}\text { 2.3.2 Bedarfsausweis zur EnEV } & 24\end{array}$

$\begin{array}{lll}2.3 .3 & \text { DIN } \vee 18599 & 24\end{array}$

2.3.4 Die Zonierung von Nichtwohngebäuden 26

2.3.5 Sonderfälle und Probleme zur Auslegung nach EnEV 28

2.3.6 Verordnung zur Umsetzung der Energieeinsparverordnung - EnEV-UVO $\quad 30$

2.4 Erneuerbare-Energien-Wärmegesetz - EEWärmeG 31

2.5 Europäische Union - Richtlinie der Gesamteffizienz von Gebäuden 31

2.6 Entwicklung der Anforderungen an den Wärmeschutz 32

2.7 Anforderungen aus der EnEV 2014: Fassung $2016 \quad 34$

$3 \quad$ Wärmeschutz: Definition und Erläuterung der wesentlichen Fachbegriffe _ $\quad 37$

$\begin{array}{lll}3.1 & \text { Energie } & 37\end{array}$

3.2 Einheiten und physikalische Größen 39

3.3 Allgemeine Begriffe der Wärmelehre $\quad 40$

3.3.1 Wärmeleitfähigkeit $\lambda \quad 41$

$\begin{array}{lll}\text { 3.3.2 Wärmedurchlasswiderstand R } & 43\end{array}$

3.3.3 Wärmeübergangswiderstand und Wind 45

3.3.4 Wärmestromdichte q, Wärmestrom und Isotherme $\quad 48$

3.3.5 U-Wert oder Wärmedurchgangskoeffizient 49

3.3.6 Der k-Wert 51

3.3.7 Wärmemenge Q oder spezifische Wärmekapazität c $\quad 51$

3.3.8 Der Wärmeeindringkoeffizient b und die Kontakttemperatur 52

$\begin{array}{ll}3.3 .9 \quad \text { Emissionsgrad } \varepsilon & 52\end{array}$

3.4 Mechanismen des Wärmetransports 54

3.4.1 Wärmestrahlung $\quad 55$

$\begin{array}{lll}3.4 .2 & \text { Konvektion } & 55\end{array}$

$\begin{array}{lll}3.4 .3 & \text { Wärmeleitung } & 56\end{array}$

$4 \quad$ Energetischer Wärmeschutz 59

4.1 Vorgaben und Gliederung der Energieeinsparverordnung $\quad 60$

4.1.1 Wohngebäude 62

$\begin{array}{lll}\text { 4.1.2 Nichtwohngebäude } & 63\end{array}$

4.1.3 Bestandsbauten und Denkmäler 63 
4.2 Die energetische Bewertung der Hüllfläche $\quad 65$

4.2.1 Transmissionswärmeverluste $\mathrm{H}_{\top} \quad 66$

4.2.2 Der Fx-Temperatur-Korrekturfaktor 67

$\begin{array}{lll}4.3 & \text { Wärmebrücken und energetische Nachweise } & 67\end{array}$

4.3.1 Randbedingungen der Wärmebrückenbetrachtungen $\quad 70$

$\begin{array}{lll}\text { 4.3.2 } & \text { Arten der Wärmebrücke } & 71\end{array}$

4.3.3 Wärmebrücken nach DIN 4108 Beiblatt $2 \quad 76$

4.3.4 Thermische Trennungen 78

4.3.5 Wärmeverluste gegen Erdreich 82

$4.4 \quad$ Luftdichtheit und Wärmeschutz 87

4.4.1 Luftdichtheitskonzept 89

4.4.2 Verarbeitung und Anschlüsse von Folien 90

4.4.3 Luftdichte Anschlüsse von Fensterkonstruktionen $\quad 91$

4.4.4 Luftdichtheit von üblichen Baumaterialien 92

4.4.5 Luftdichtheitsmessungen nach »Blower-Door-Messverfahren« $\quad 94$

4.5 Thermografie $\quad 95$

$5 \quad$ Rechenverfahren und Nachweise 99

5.1 Beispiele zu U-Wert-Berechnungen 101

$6 \quad$ Hygienischer Wärmeschutz $\quad 107$

6.1 Schimmelpilz und Bakterien im Innenraum $\quad 107$

6.1.1 Grundlagen zur Bewertung $\quad 110$

6.1.2 Grenzwerte von Oberflächentemperaturen 111

6.1.3 Vermeidung von Schimmelwachstum 111

6.1.4 Sporengehalt, Indikatoren und gesundheitliche Risiken 112

$\begin{array}{lll}6.1 .5 & \text { Bewertung des Befalls } & 113\end{array}$

$\begin{array}{lll}\text { 6.1.6 Ablauf der Untersuchungen } & 114\end{array}$

6.1.7 Einfluss des pH-Werts auf das Schimmelpilzwachstum $\quad 115$

$\begin{array}{lll}6.1 .8 & \text { Sanierungsmaßnahmen } & 116\end{array}$

6.1.9 Rechnerische Nachweise nach DIN 4108-2 117

6.1.10 Sorptionsfähigkeit von Baustoffen - Die Ausgleichsfeuchte 119

6.1.11 Nutzerverhalten und Raumluftfeuchte $\quad 120$

$\begin{array}{ll}\text { 6.1.12 Lüftungskonzepte nach DIN 1946-6 } & 124\end{array}$

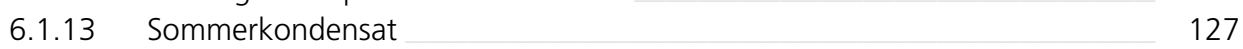

$\begin{array}{lll}6.2 & \text { Behaglichkeit } & 128\end{array}$

$\begin{array}{ll}\text { 6.2.1 Luftfeuchtigkeit und Behaglichkeit } & 130\end{array}$

6.2.2 Wärmeableitung über den Fußboden 131

6.2.3 Asymmetrische Strahlungstemperatur 132

6.2.4 Zugerscheinung an Wänden 133

6.2.5 Der Prozentsatz der Unzufriedenen (PPD) 133

$7 \quad$ Entscheidungskriterien bei der Beurteilung von Schimmelpilzschäden $\quad 135$ 
$8 \quad$ Feuchteschutz 143

8.1 Feuchteschutz nach DIN 4108-3 144

8.2 Feuchteschutz nach DIN EN ISO $13788 \quad 146$

8.3 Grundlagen zum Glaser-Nachweis 146

$\begin{array}{ll}\text { 8.3.1 } & \text { Randbedingungen Winter und Sommer } \\ 8.37\end{array}$

8.3.2 Beispiele 148

8.3.3 Feuchteschutz und Tauwasser 151

8.3.4 Trocknungsverhalten 152

8.4 Schutz der Konstruktion $\quad 154$

8.4.1 Die Wasserdampfdiffusionswiderstandszahl $\mu \quad 155$

$\begin{array}{lll}8.4 .2 & \mathrm{sd}_{\mathrm{d}} \text {-Wert } & 157\end{array}$

8.5 Konstruktionen und Nachweise 158

8.5.1 Nachweisfreie Wandkonstruktionen 159

$\begin{array}{lll}\text { 8.5.2 Nachweisfreie Bodenplatten } & 160\end{array}$

8.5.3 Nachweisfreie Dachkonstruktionen 160

8.5.4 Feuchteschutz und Fenster 161

8.5.5 Feuchteschutznachweise bei Gründächern 161

8.6 Hinterlüftete Bauteile 163

8.7 Feuchteschutz bei Holzkonstruktionen 163

8.8 Feuchteschutz bei Stahlkonstruktionen 166

8.9 Mechanismen der Aufnahme von Feuchtigkeit 167

$\begin{array}{lll}8.9 .1 & \text { Adsorption } & 169\end{array}$

$\begin{array}{lll}8.9 .2 & \text { Absorption } & 169\end{array}$

$\begin{array}{lll}\text { 8.9.3 Desorption } & 170\end{array}$

$\begin{array}{lll}\text { 8.9.4 Ausgleichsfeuchte } & 171\end{array}$

$9 \quad$ Sommerlicher Wärmeschutz 173

9.1 Nachweispflicht nach DIN 4108-2 173

$\begin{array}{ll}9.2 \text { Nachweisfreie Räume } & 175\end{array}$

9.3 Grundlagen der Berechnung 175

$\begin{array}{ll}\text { 9.3.1 Sommerklimaregionen } & 176\end{array}$

$\begin{array}{ll}\text { 9.3.2 Sonneneintragskennwert s } & 177\end{array}$

$\begin{array}{ll}\text { 9.3.3 Gesamtenergiedurchlass gtot } & 178\end{array}$

$\begin{array}{ll}\text { 9.3.4 Sonnenschutzverglasung } & 179\end{array}$

9.3.5 Abminderungsfaktor FC und Teilbestrahlungsfaktor FS 180

$\begin{array}{ll}\text { 9.3.6 Ausrichtung des Gebäudes } & 181\end{array}$

9.3.7 Konstruktionsart und nächtliches Lüften 182

9.3.8 Beispiel: Berechnung Sonneneintragskennwert 186

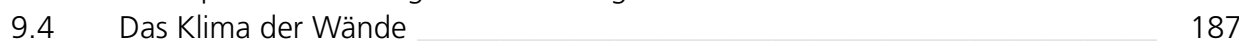

9.4.1 Individuelles Wohlbefinden 188 
10 Typische bauliche Problemfelder und Schadensbilder 189

10.1 Grundlagen zur Dämmung 189

10.2 Außendämmung 190

10.2.1 Algen-, Pilzbefall und gedämmte Fassaden 191

$\begin{array}{lll}10.2 .2 & \text { Algen und Pilze } & 194\end{array}$

$\begin{array}{lll}10.2 .3 & \text { Biozide } & 195\end{array}$

$\begin{array}{ll}\text { 10.2.4 Dämmung und Brandschutz } & 195\end{array}$

$\begin{array}{ll}10.3 \text { Innendämmung } & 198\end{array}$

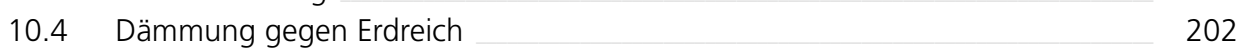

$\begin{array}{lll}10.5 & \text { Solare Erwärmung von Bauteilen } & 203\end{array}$

10.6 Maßnahmen gegen Feuchtigkeit 208

$\begin{array}{lll}\text { 10.6.1 Baufeuchte } & 208\end{array}$

$\begin{array}{ll}\text { 10.6.2 Ausgleichsfeuchte } & 211\end{array}$

$\begin{array}{ll}\text { 10.6.3 Porensysteme und ihre Eigenschaften } & 212\end{array}$

$\begin{array}{ll}\text { 10.6.4 Mauerwerk } & 214\end{array}$

$\begin{array}{lll}10.6 .5 & \text { Porenbeton } & 215\end{array}$

10.6.6 Auswirkungen der Trocknungsprozesse auf Holzbauteile 215

11 Zukünftige Anforderungen und nachhaltiger Wärmeschutz 219

12 Quellen, Abbildungen, Sachregister 223

$\begin{array}{ll}12.1 & \text { Literatur } \\ 12.2 & 223\end{array}$

$\begin{array}{ll}12.2 & \text { Normen und Verordnungen } \\ 12.326\end{array}$

$\begin{array}{ll}12.3 & \text { Abbildungen } \\ & 228\end{array}$

$\begin{array}{lll}12.4 & \text { Akronyme } & 229\end{array}$

$\begin{array}{ll}12.5 & \text { Sachregister } \\ & 230\end{array}$ 